\title{
Oesophageal mesenchymal tumours: clinicopathological features and absence of Epstein-Barr virus
}

King Y Lam

\begin{abstract}
Background-Recent studies have suggested that the Epstein-Barr virus (EBV) is associated with smooth muscle tumours (leiomyoma and leiomyosarcoma) in patients with human immunodeficiency virus and in organ transplant recipients. Leiomyoma is the most common mensenchymal tumour found in the oesophagus. Aim-To report a single institution experience on oesophageal mesenchymal tumours and to determine whether EBV is associated with these tumours.

Methods-40 sporadic oesophageal mesenchymal tumours were studied and their diagnosis confirmed on pathological review and immunohistochemical studies. Formalin fixed, paraffin wax embedded tissues from these tumours were analysed for EBV using in situ hybridisation for two messenger RNA (mRNA) probes, EBER and BamH1 W.
\end{abstract}

Results-The oesophageal mesenchymal tumours comprised 36 leiomyomas, two undifferentiated stromal tumours, and two gastrointestinal autonomic nerve tumours (GANTs). Median age of the patients with leiomyoma $(26$ men, 10 women) was 62 years (range 30 to 85 ) and $81 \%$ of them had an asymptomatic lesion. The median longitudinal size was $1.2 \mathrm{~cm}$. Multiple leiomyomas were seen in $11 \%$ of the patients and calcification was noted in one tumour. Coexisting squamous cell carcinoma was found in one third of cases. The stromal tumours were small, asymptomatic, and located in the lower third of the oesophagus, while the GANTs were large, symptomatic, and found in the upper third of the oesophagus. EBV mRNAs were not detected in all these tumours.

Conclusions-The clinicopathological features of oesophageal leiomyoma, undifferentiated stromal tumour, and GANT were different. Some oesophageal leiomyomas were associated with oesophageal squamous cell carcinomas. EBV is not associated with sporadic oesophageal mesenchymal tumours.

(F Clin Pathol 1999;52:758-760)

Keywords: leiomyoma; Epstein-Barr virus; stromal tumour; autonomic nerve tumour

Recent studies suggest that Epstein-Barr virus (EBV) is a risk factor for the development of smooth muscle tumours (leiomyoma or leio- myosarcoma) in immunocompromised patients such as those affected by AIDS and in organ transplant recipients. ${ }^{1-12}$ The smooth muscle tumours are believed to originate from the clonal expansion of individual smooth muscle cells infected with EBV. EBV shows tropism for lymphoid and epithelial cells, not smooth muscle cells. ${ }^{13}$ Reactivation of EBV infection is enhanced during immunosuppression and this may lead to dissemination of EBV to tissue that is not normally infected by EBV, such as smooth muscle. ${ }^{14}$

Stromal tumours of the gastrointestinal tract can be broadly defined as mesenchymal neoplasms originating from the muscle wall of hollow viscera. Most show total lack or incomplete features of smooth muscle or neural differentiation. ${ }^{15}$ In a minority of cases only, mainly confined to the oesophagus and rectum, are the tumours composed mainly of smooth muscle. In the oesophagus, leiomyomas account for more than $50 \%$ of benign oesophageal tumours. ${ }^{16}$ Nonetheless, the characteristics of oesophageal leiomyomas have received little attention in published reports.

In this study, the clinicopathological features of patients with oesophageal mesenchymal tumours collected over a 15 year period in a referral centre for oesophageal surgery were analysed. In addition, the presence of EBV in these tumours was studied to determine whether the virus is involved in the pathogenesis of sporadic oesophageal mesenchymal tumours.

\section{Methods}

COLLECTION OF DATA

The pathology records from the Queen Mary Hospital were analysed over a 15 year period (1983 to 1997) to identify cases with oesophageal mesenchymal tumours. The age, sex, and clinical presentation of the patients were recorded, as well as the location and longitudinal extent of the tumours. Histological sections of these cases were retrieved and reviewed. A paraffin block was chosen for immunohistochemical studies and in situ hybridisation.

\section{IMMUNOHISTOCHEMICAL STUDIES}

Five micron paraffin sections from the paraffin blocks were deparaffinised and rehydrated. Immunohistochemical studies were performed using the avidin-biotin-peroxidase complex method. Appropriate negative controls (using buffer solution instead of primary antibodies) and positive controls from normal tissues were also used. Antibodies to desmin (monoclonal, 
1:50, microwave pretreated, Dakopatts), actin (monoclonal, 1:200, Dakopatts), S-100 (polyclonal, 1:200, Dakopatts), and vimentin (monoclonal, 1:20, microwave pretreated, Dakopatts) were applied to all cases. Avidin-biotin complex and diaminobenzidine (DAB) were used as the detection system. Brown staining was recorded as positive.

\section{IN SITU HYBRIDISATION}

Five micron paraffin sections from the paraffin blocks were deparaffinised, rehydrated, and pretreated with proteinase K (Sigma). In situ hybridisation for EBV encoded RNA (EBER) and BamHI W was then performed on these sections. EBER probe is specific for EBER mRNA transcripts (EBER1 and 2), expressed in high copy numbers during the latent phase of the EBV life cycle. The probe is targeted at the nucleotides 6629 to 7128 of EBV. BamHI $\mathrm{W}$ is a DNA sequence reiterated 11 times within the EBV viral genome. The probe was obtained from Kreatech Diagnostic (Amsterdam). Non-radioactive methods were used, as described previously. ${ }^{17}$ Nitro-blue tetrazolium and 5-bromo-4-chloro-3-indolyphophate (BCIP) were used as substrate. Cell lines and nasopharyngeal carcinoma known to be positive for EBV were used as positive controls. RNase A treated serial sections and hybridisation buffer without probe were used as negative controls. Sections were counterstained with methyl green. The slides were then mounted and examined. Positive staining was recognised as black coloration at the site of hybridisation.

\section{Results}

Forty patients (27 men, 13 women) with oesophageal mesenchymal tumours were found. Thirty six patients had leiomyomas, two with stromal tumours and two with gastrointestinal autonomic nerve tumours (GANTs).

The leiomyomas were benign smooth muscle tumours characterised by positive staining to desmin and actin but negative to S-100. GANTs were mesenchymal tumours of borderline malignant potential defined by ultrastructural and immunohistochemical stud-

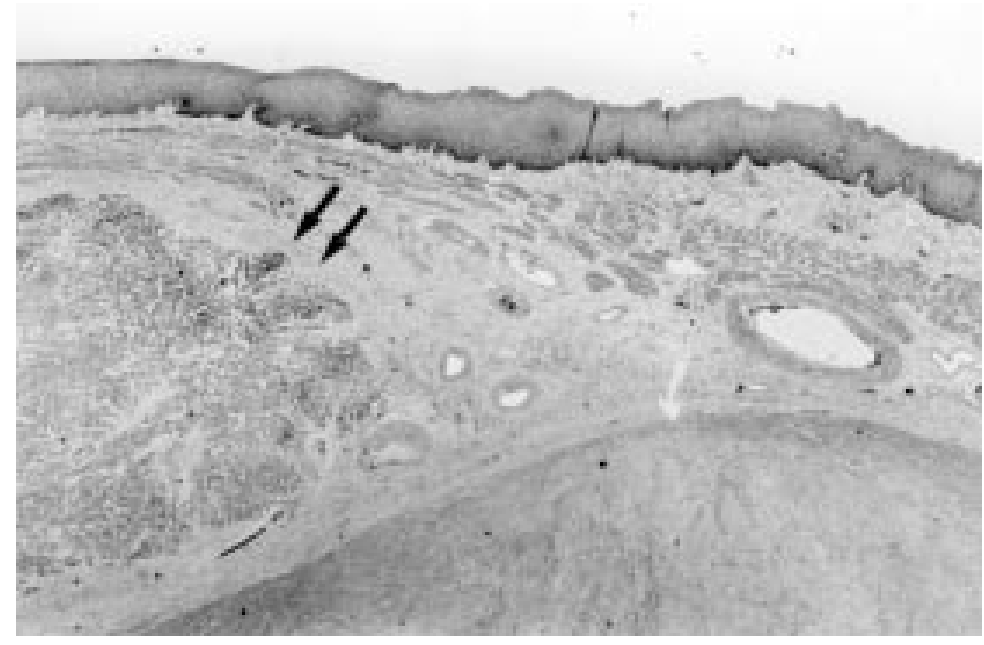

Figure 1 Calcification (arrows) noted in oesophageal leiomyoma. (haematoxylin and eosin, $\times 81$. ies, as reported in our previous study. ${ }^{18}$ They were large (diameter $6.5 \mathrm{~cm}$ and $8 \mathrm{~cm}$, respectively), symptomatic, and found in the upper third of the oesophagus.

Stromal tumours were undifferentiated mesenchymal tumours (positive for vimentin) that showed no evidence of smooth muscle differentiation (negative to desmin and actin). The stromal tumours were found in a 50 year old man and a 66 year old woman. The tumours were small (each had a diameter of $0.3 \mathrm{~cm}$ ), asymptomatic, and located in the lower third of the oesophagus.

The GANTs were larger (diameter $6.5 \mathrm{~cm}$ and $8 \mathrm{~cm}$, respectively), symptomatic, and found in the upper third of the oesophagus.

The median age of the 36 patients with leiomyomas (26 men, 10 women) was 62 years (range 30 to 85 ). Most of these patients were asymptomatic ( $81 \%$ asymptomatic, $n=29)$, while $19 \%(n=7)$ presented with dysphagia. The patients with asymptomatic tumours were older than those with symptomatic tumours (mean age $62 v 48$ years, $\mathrm{p}=0.02$ by Mann-Whitney $U$ test). The tumours were often located in the middle third of the oesophagus $(25 \%, \mathrm{n}=9$, in the upper third; $50 \%, \mathrm{n}=18$, in the middle third; $25 \%, \mathrm{n}=9$, in the lower third).

The median diameter of the leiomyomas was $1.2 \mathrm{~cm}$ (range, 0.1 to $7 \mathrm{~cm}$ ). Symptomatic tumours were slightly larger than asymptomatic tumours (mean diameter $3.2 \mathrm{~cm} v 1.3$ $\mathrm{cm}, \mathrm{p}=0.06$ by Mann-Whitney U test). On histological examination, $75 \%(n=27)$ of the leiomyomas were derived from the muscle layers and $25 \%(n=9)$ from the muscularis mucosae. Multiple leiomyomas were seen in $11 \%$ of the patients (four of 36). In three of the four cases, two leiomyomas were present. The remaining case had five leiomyomas in the oesophagus. One of the 36 patients had calcification in the leiomyoma (fig 1). Coexisting squamous cell carcinoma of the oesophagus was found in 12 patients.

None of the 40 mesenchymal tumours was positive for any of the two probes for EBV. The lymphocytes, oesophageal mucosa, and smooth muscle were also negative for EBV. On the other hand, strong signals were observed in the nuclei of the tumour cells in the control tissues.

\section{Discussion}

This series represents one of the largest single institution experiences of oesophageal mesenchymal tumours. In this study, leiomyoma was the most common mesenchymal tumour noted in the oesophagus. In addition, four unusual oesophageal mesenchymal tumours (two GANTs and two undifferentiated stromal tumours) were found. The features of the two GANTs have been described in our previous report. ${ }^{18}$ The diagnosis of undifferentiated oesophageal stromal tumours was based on both morphology and immunohistochemistry. Although these tumours are common in other parts of the gastrointestinal tract, they have never been properly characterised and described in the oesophagus. In the present study, the tumours were found to be small (diameter 
of each around $0.3 \mathrm{~cm}$ ), asymptomatic, and located in the lower third of the oesophagus. On the other hand, the GANTs were large (diameter 6.5 and $8.0 \mathrm{~cm}$, respectively), symptomatic, and occurred in the upper third of the oesophagus.

Oesophageal leiomyomas occur in both sexes, but there is a male preponderance. ${ }^{16}$ Both multiple leiomyomas and calcification in an oesophageal leiomyoma are said to be uncommon. In the present study, the sex ratio of 2.6 to 1 was similar to that reported in other studies. ${ }^{16}$ Calcification was uncommon, being found in only one case; however, multiple tumours were seen in $11 \%$ of the patients.

The coexistence of leiomyoma and squamous cell carcinoma in the oesophagus is uncommon and has been the subject of many case reports. ${ }^{1925}{ }^{25}$ Less than 30 cases have been reported in all. In the present study, one third of the patients with leiomyoma had concurrent squamous cell carcinomas. The high incidence of co-occurrence of these two tumours suggests this is unlikely to be coincidental. One tumour may create an environment that directly or indirectly favours the genesis of the second tumour. For example, oesophageal stenosis produced by either a leiomyoma or a carcinoma might induce or promote the formation of the other tumour.

The close proximity of the oesophagus to the nasopharynx and the recent detection of EBV in smooth muscle tumours of immunocompromised patients raise the possibility that EBV may be involved in the pathogenesis of oesophageal mesenchymal tumours. In our previous investigation, EBV was not detected in 74 oesophageal squamous cell carcinomas but was noted in a few lymphocytes adjacent to the tumour epithelia. ${ }^{27}$ In the current study, EBV was not detected in 36 oesophageal leiomyomas and two stromal tumours. Although the two oesophageal GANTs showed prominent lymphoid infiltrates, EBV was not noted in the tumour cells and the lymphoid infiltrates.

EBV has been linked to smooth muscle tumours in immunocompromised patients. The smooth muscle tumours developed in these patients were often located in the liver and lung and sometimes in spleen and adrenal gland. ${ }^{1-12}$ In gastrointestinal tract, these EBV related smooth muscle tumours have been reported in stomach and in large and small intestine. To our knowledge, no such case has been reported in the oesophagus. This may imply that oesophageal smooth muscle is resistant to EBV induced proliferation. The data in the current study suggest that EBV is unlikely to be an important aetiological factor in oesophageal smooth muscle tumours in immunocompetent individuals. Although it could be argued that EBV is present at a level below the detection threshold of in situ hybridisation, false negative results are unlikely as two highly sensitive probes to EBV (EBER and BamH1W) and positive control tissues were used.
I would like to acknowledge Dr Gopesh Srivastava and his staff for their technical assistance

1 Bluhm JM, Yi ES, Diaz G, et al. Multicentric endobronchial smooth muscle tumors associated with the Epstein-Barr smooth muscle tumors associated with the Epstein-Barr
virus in an adult patient with the acquired immunovirus in an adult patient with the acquired immunodeficie.

2 Boman F, Gultekin H, Dickman PS. Latent Epstein-Barr virus infection demonstrated in low-grade leiomyosarcomas of adults with acquired immunodeficiency syndrome, but not in adjacent Kaposi's lesion or smooth muscle tumors in immunocompetent patients. Arch Pathol Lab Med 1997;121:834-8.

3 de-Chadarevian JP, Wolk JH, Inniss S, et al. A newly recognized cause of wheezing: AIDS-related bronchial leiomyomas. Pediatr Pulmonol 1997;24:106-10.

4 Morgello S, Kotsianti A, Gumprecht JP, et al. Epstein-Barr virus-associated dural leiomyosarcoma in a man infected with human immunodeficiency virus: case report. 7 Neurosurg 1997;86:883-7.

5 Davidoff AM, Hebra A, Clark BJ, et al. Epstein-Barr virusassociated hepatic smooth muscle neoplasm in a cardiac transplant recipient. Transplantation 1996;61:515-17.

6 Kleinschmidt-DeMasters BK, Mierau GW, Sze CI, et al. Unusual dural and skull-based mesenchymal neoplasms: a report of four cases. Hum Pathol 1998;29:240-5.

7 Jenson HB, Leach CT, McClain KL, et all. Benign and malignant smooth muscle tumors containing Epstein-Barr virus in children with AIDS. Leuk Lymphoma 1997;27:30314.

8 Jimenez-Heffernan JA, Hardisson D, Palacios J, et al. Adrenal gland leiomyoma in a child with acquired immunodeficiency syndrome. Pediatr Pathol Lab Med 1995;15:923-9.

9 Timmons CF, Dawson DB, Richards CS, et al. Epstein-Barr virus-associated leiomyosarcomas in liver transplantation recipients. Origin from either donor or recipient tissue. Cancer 1995;76:1481-9.

10 Zetler PJ, Filipenko JD, Bilbey JH, et al. Primary adrenal leiomyosarcoma in a man with acquired immunodeficiency syndrome (AIDS): further evidence for an increase in smooth muscle tumors related to Epstein-Barr infection in AIDS. Arch Pathol Lab Med 1995;119:1164-7.

11 McClain KL, Leach CT, Jenson HB, et al. Association of Epstein-Barr virus with leiomyosarcomas in children with AIDS. N Engl f Med 1995;332:12-18.

12 Prevot S, Neris J, de-Saint-Maur PP. Detection of Epstein Barr virus in an hepatic leiomyomatous neoplasm in an adult human immunodeficiency virus 1-infected patient. Virchows Arch 1994;425:321-5.

13 Brooks L, Thomas JA. The significance of Epstein-Barr virus in the pathogenesis of lymphoma and epithelial neoplasia. Curr Diagn Pathol 1995;2:163-74

14 Liebowitz D. Epstein-Barr virus-an old dog with new tricks. N Engl f Med 1995;332:55-67.

15 Miettinen M. Gastrointestinal stromal tumors: an immunohistochemical study of cellular differentiation. Am $\mathcal{F}$ Clin Pathol 1988;89:601-10.

16 Seremetis MG, Lyons WS, DeGuzman VC, et al. Leiomyomata of the esophagus: an analysis of 838 cases. Cancer 1976;38:2166-77

17 Tao Q, Ho FC, Loke SL, et al. Epstein-Barr virus is localized in the tumor cells of nasal lymphomas of NK, T or B-cell type. Int $\mathcal{F}$ Cancer 1995;60:315-20.

18 Lam KY, Law SYK, Chu KM, et al. Gastrointestinal autonomic nerve tumor of the esophagus: a clinicopathologic, immunohistochemical, ultrastructural study of a case and review of the literature. Cancer 1996;78:1651-9.

19 Visioli A, Daniel FJ. Leiomyosarcoma of the esophagus: a case report and literature review of leiomyosarcoma. Aust Radiol 1997;41:160-5.

20 Takubo K, Nakagawa H, Tsuchiya S, et al. Seedling leiomyoma of the esophagus and esophagogastric junction zone. Hum Pathol 1981;12:1006-10.

21 Iizuka T, Kato H, Watanabe G, et al. Superficial carcinoma of the esophagus coexisting with esophageal leiomyoma: a case report and review of the Japanese literature. fpn f Clin Oncol $1984 ; \mathbf{1 4}: 115-12$.

22 Watanabe M, Baba T, Hotchi M. A case of leiomyoma of the lamina muscularis mucosae of the esophagus with a complication of carcinoma in situ of the overlying mucosa. Acta Pathol fpn 1987;37:1845-51.

23 Sarbia M, Katoh E, Borchard F. Collision tumor of squamous cell carcinoma and leiomyoma in the esophagus. Path Res Pract 1993;189:360-2.

24 Yoshikane H, Tsukamoto Y, Niwa Y, et al. The coexistence of esophageal submucosal tumor and carcinoma. Endoscopy 1995;27:119-23.

25 Nagashima R, Takeda H, Motoyama T, et al. Coexistence of superficial esophageal carcinoma and leiomyoma: case report of an endoscopic resection. Endoscopy 1997;29:683-4.

26 Pai SA, Kher N, Krishnamurthy S. Collision tumor with three components at esophagogastric junction. Indian $\mathcal{F}$ Gastroenterol 1997;16:116.

27 Lam KY, Srivastava G, Leung ML, et al. Absence of Epstein-Barr virus in esophageal squamous cell carcinoma: a study of 74 cases using in-situ hybridization. 7 Clin Pathol Mol Pathol 1995;48:M188-90. 